\section{Paciente con vértigo, situación frustrante para el médico y el paciente}

\section{Sr. Director:}

La valoración del paciente con vértigo, una de las molestias más comunes por la que se busca atención médica, a menudo es estimulante y frustrante para el médico. Etimológicamente, la palabra vértigo viene de la raíz latina vertere, que significa girar. El vértigo es una ilusión de movimiento, síntoma complejo que puede tener muchas causas. Resulta difícil valorar la magnitud del vértigo, pues se trata de una molestia subjetiva que no puede medirse. La anamnesis y la exploración física establecerán en qué categoría se define mejor a cada enfermo: si es vértigo verdadero, presíncope, desequilibrio o aturdimiento. Una vez averiguado esto, se puede pautar el tratamiento apropiado, y producir resultados gratificantes para el paciente y el clínico.

Entre las personas con vértigo que buscan atención, casi $70 \%$ son atendidas al inicio por internistas generales o médicos familiares. Los vértigos se dan en el $1 \%$ de la población, que motivan el $15 \%$ de las consultas neurológicas atendidas por el médico de Atención Primaria (1).

Hoy día los Servicios de Urgencias están cada vez más saturados y con una alta demanda asistencial ello conlleva que en muchas ocasiones no se disponga del tiempo necesario para ponerle nombre y apellido o por lo menos aproximarnos al diagnóstico del cuadro vertiginoso que tiene cada enfermo y simplemente son derivados a los Servicios de Otorrinolaringología con la petición de "para mejor estudio y tratamiento".

Lo primero que tenemos que descartar en estos casos es si la causa es central o periférica. La principal utilidad de la valoración neurológica general antes de medicar al enfermo es buscar indicadores o signos clínicos de que el tallo encefálico u otros sitios del sistema nervioso central (SNC) son la causa de la molestia del enfermo. Las alteraciones originadas en el (SNC) se encuentran a menudo en la exploración de los pares craneales. El inicio gradual sugiere origen en el SNC, los síntomas intermitentes son típicos de enfermedad del oído interno (OI) y los síntomas continuos indican que es más probable el trastorno del SNC. En cuanto a la duración como regla, entre más central el proceso, es más prolongado (2).

El diagnóstico de vértigo periférico se confirma con un signo característico en la exploración, el nistagmus. La característica es que sea horizontorrotatorio espontáneo hacia el lado dominante (oído sano). Lo segundo a buscar son las desviaciones corporales y segmentarias hacia el lado lesionado (3-5).

Por orden de frecuencia las posibilidades diagnósticas del vértigo periférico agudo es el vértigo posicional paroxístico benigno (VPPB), seguido de la neuronitis vestibular y, a su vez esta es la primera causa en personas jóvenes.

Existen cuatro grandes causas de vértigo periférico: las ya mencionadas, el síndrome de Meniére y la cervicoartrosis, aunque son muy pocos los médicos que aceptan que la cervicoartrosis sea una causa de vértigo y de mareos. Más bien son los llamados drop-attacks o episodios de insuficiencia en la vascularización vertebrobasilar, en los que podría asociarse, junto a otros síntomas neurológicos, una sensación de vértigo con los movimientos de la cabeza.

El período vertiginoso puede durar entre 5-20 días, siendo gradualmente menor debido a la compensación central, que puede llegar a durar otras tres semanas donde el paciente referirá inestabilidad, desequilibrio e inseguridad. La compensación es mucho más rápida y completa en pacientes jóvenes que en ancianos. Por la particularidad de compensación que tiene el aparato vestibular cuando llegan a ser valorado por el ORL ya el cuadro ha desapa- recido pero los pacientes refieren que ya que tienen dada la cita vienen a consulta (5-7).

Queremos hacer especial hincapié que en la fase aguda hay que usar sedantes vestibulares y antieméticos mientras el paciente tenga náuseas y/o vómitos que dificulten la realización de su vida habitual. Lo más importante es retirar los sedantes vestibulares lo más precozmente al segundo o tercer día, manteniendo o no la medicación antiemética. Mientras más pronto se le diga al paciente que inicie la rehabilitación vestibular antes se recuperará.

La tendencia natural del paciente es evitar mover la cabeza y otros tipos de movimientos por temor a exacerbar los síntomas. La falta de movilidad de la cabeza puede, de hecho, prolonga la duración de los síntomas. En otros, la inactividad forzada origina desacondicionamiento físico además de debilidad muscular y cambios ortostáticos.

Lamentablemente seguimos recibiendo pacientes con largas esperas para ser valorado por el ORL, sometidos a varios meses de tratamiento antivertiginoso y tienen una neuronitis vestibular o un VPPB. Lo habitual es que lleguen en busca del ansiado estudio de imagen tipo "scanner de la cabeza" (8).

Si en médico generalista o de cabecera se apoya de diferentes tablas sencillas de ejercicios publicadas para tratar al paciente vertiginoso, de seguro lograremos recuperar a muchos enfermos que por temor a enfrentarse a su realidad optan por el reposo y su médico por la medicación $(3,9,10)$.

\section{A. M. García de Hombre}

Servicio de Otorrinolaringología. Hospital General de Fuerteventura

1. Herdman S, Whitney S. Valoración del tratamiento fisioterapéutico de la hipofunción vestibular. En: Suárez C, Gil-Carcedo, Marco J, Medina J, Ortega P, Trinidad T, eds. Tratado de Otorrinolaringología y Cirugía de Cabeza y Cuello. Madrid: Proyectos Médicos S.L, 1999; 1599-1622.

2. Swartz R, Longwell P. Treatment of vertigo. Am Fam Physician 2005; 71: 1115-22.

3. Yardley L, Donovan-Hall M, Smith HE, Walsh BM, Mullee M, Bronstein AM. Effectiveness of primary care-based vestibular rehabilitation for chronic dizziness. Ann Intern Med 2004; 141: 598-605.

4. Prokopakis EP, Chimona T, Tsagournisakis M, Christodoulou P, Hirsch BE, Lachanas VA, Helidonis ES, Plaitakis A, Velegrakis GA. Benign paroxysmal positional vertigo: 10-year experience in treating 592 patients with canalith repositioning procedure. Laryngoscope 2005; 115 : 1667-71.

5. Godemann F, Siefert K, Hantschke-Bruggemann M, Neu P, Seidl R, Strohle A. What accounts for vertigo one year after neuritis vestibularisanxiety or a dysfunctional vestibular organ? J Psychiatr Res 2005; 39: 529-34.

6. Herdman SJ. Role of vestibular adaptation in vestibular rehabilitation. Otolaryngol. Head Neck Surg 1998; 119: 49-54.

7. Radtke A, von Brevern M, Tiel-Wilck K, Mainz-Perchalla A, Neuhauser H, Lempert. Self-treatment of benign paroxysmal positional vertigo: Semont maneuver vs Epley procedure. Neurology 2004; 63: 150-2.

8. Barona de Guzman R, Armengot Carceller M. Vestibular compensation. Rehabilitation of a patient with vertigo. Acta Otorrinolaringol Esp 1994; 45: 401-5.

9. Epley JM. The canalith reposition procedure for treatment of BPPV. Otolaryngol Head Neck Surgery 1992; 32: 290-296

10. Semont A, Freyss G, Vitte E. Curing The VPPV with a liberatory maneuver. Adv Otorhinolaryngol 1988; 32: 390-396. 